\title{
Risk factors and prognostic influence of infection in a single cohort of 87 adults with systemic lupus erythematosus
}

\author{
V Noël, O Lortholary, P Casassus, P Cohen, T Généreau, M-H André, L Mouthon, \\ L Guillevin
}

Fédération de

Médecine Interne, Maladies Infectieuses et Tropicales, Hôpital Avicenne, Université Paris-Nord, Bobigny, France

V Noël

O Lortholary

P Casassus

P Cohen

T Généreau

M-H André

L Mouthon

L Guillevin

Correspondence to: Professor L Guillevin,

Hôpital Avicenne, 125, route de Stalingrad, 93009

Bobigny, France

loic.guillevin@

avc.ap-hop-paris.f

Accepted 26 April 2001

This work was presented in part at the 38th Interscience Conference on Antimicrobial Agents and Chemotherapy, San Diego, California, USA, 1998 (abstract L-61), and at the Meeting of the American College of Rheumatology, Boston, Massachusetts, November 1999 (abstract 174).

\begin{abstract}
Objectives-To describe infectious complications and analyse their risk factors and prognostic role in adults with systemic lupus erythematosus (SLE).

Methods-A monocentric cohort of 87 adults with SLE (1960-1997) was studied to determine the risk factors for infection (disease activity evaluated by SLAM and SLEDAI scores, type of organ(s) involved or any biological abnormality, specific treatments) by comparing patients who had suffered at least one infectious episode $(n=35 ; 40 \%)$ with non-infected patients $(n=52 ; 60 \%)$. Prognostic indicators were assessed by comparing survivors at 10 years with non-survivors.

Results-Of the 57 infectious episodes, 47 $(82 \%)$ were of bacterial origin, $16(28 \%)$ were pneumonia, and $46(81 \%)$ were community acquired. According to univariate analysis, significant risk factors for infection were: severe flares, lupus glomerulonephritis, oral or intravenous corticosteroids, pulse cyclophosphamide, and/or plasmapheresis. No predictors were identified at the time of SLE diagnosis. Multivariate analyses retained intravenous corticosteroids $(p<0.001)$ and/or immunosuppressants $(p<0.01)$ as independent risk factors for infection, which was the only factor for death after $\mathbf{1 0}$ years of evolution $(\mathrm{p}<0.001)$.

Conclusion-In adults with SLE, infections are common and most often caused by community acquired bacteria. Intravenous corticosteroids and immunosuppressants are independent risk factors for infection, which is the only independent risk factor for death after 10 years of SLE evolution.

(Ann Rheum Dis 2001;60:1141-1144)
\end{abstract}

Systemic lupus erythematosus (SLE) is an autoimmune disease which primarily affects young people, and can cause death in up to $50 \%$ of them. ${ }^{1-3}$ In large clinical series of patients with SLE, infectious complications occurred in $14-45 \%$ of them. ${ }^{13}$ The role of SLE itself and the consequences of its immunosuppressive treatments in the induction of infection remain to be elucidated.

From a methodological point of view, previous studies did not evaluate predictors of infection at the time of SLE diagnosis or immediately before the infectious event. Moreover, an overall analysis of SLE evolution from the time of diagnosis, including SLE activity scores at diagnosis, has never been undertaken to evaluate infectious consequences. In addition, the prognostic impact of infections on patients with SLE remains a matter of debate. ${ }^{34}$

The aims of this study were to describe infectious complications and analyse their risk factors and prognostic influence using a single cohort of 87 adults with SLE.

\section{Patients and methods}

PATIENTS

We retrospectively analysed the medical charts of all adults (over 16 years old) who met the American Rheumatism Association criteria for $\mathrm{SLE}^{5}$ and who were seen at least once a year (outpatient visits or admission to hospital) by the same team between 1 January 1960 and 30 September 1997.

Two groups of patients were established: those who experienced at least one infectious complication throughout their entire SLE evolution and who were admitted to hospital or examined at least once by one of us during the infectious episode; and those who had no relevant infectious episodes and did not require a hospital consultation or admission. Pertinent clinical and biological information (differential white blood cell count, CD4 T lymphocytes as of 1991, haemoglobin, platelet count, erythrocyte sedimentation rate, serum $\mathrm{C}$ reactive protein concentration, serum creatinine, protein fractions, antinuclear antibodies, anti-native DNA antibodies, C3, C4, $\mathrm{CH}_{50}$, urine analysis) was collected at diagnosis, at the time of each SLE flare, and during each infectious episode ${ }^{6}$; in addition, similar data obtained as close as possible before each infectious episode were recorded. All microbiological data available for each infectious episode were also noted. Specific SLE treatments were also recorded.

METHODS

Using two validated scores, the Systemic Lupus Activity Measure (SLAM) and the Systemic Lupus Evaluation Disease Activity Index (SLEDAI), SLE activity was evaluated at diagnosis, as near as possible before infection, and during the infectious episode. For all patients, the highest disease activity scores recorded during evolution were retained for analysis. An SLE flare was defined as the occurrence and/or aggravation of clinical manifestations directly attributable to SLE in the absence of any alternative cause. SLE glomerulonephritis was suspected in the presence of proteinuria $(>500$ 
$\mathrm{mg} / 24 \mathrm{~h}$ ) with or without renal insufficiency (creatininaemia $>120 \mu \mathrm{mol} / \mathrm{l}$ ) and was always confirmed histologically. ${ }^{6}$ Classically defined central nervous system and heart involvement was also recorded. ${ }^{6}$ Haematological flares were defined as the occurrence of autoimmune haemolytic anaemia or immunological thrombocytopenia.

When SLE treatment was considered, oral corticosteroids were taken into account when given for at least eight days at a minimal daily dose of $0.5 \mathrm{mg} / \mathrm{kg}$. Cumulative doses - that is, the mean daily dose multiplied by the number of days of treatment-were calculated as described by Stuck et al. ${ }^{7}$ Similar analyses were performed for patients who received intravenous steroids ( $\geqslant 15 \mathrm{mg} / \mathrm{kg}$ methylprednisolone) including the number of infusions, and also for patients given other immunosuppressive drugs (oral or pulse cyclophosphamide, azathioprine, or methotrexate). The number of plasmapheresis sessions and the type of intravenous catheters used were also noted. Univariate and multivariate analyses of risk factors for infection were performed by taking into account the total number of infectious episodes.

Infectious episodes were categorised as those requiring admission to our department or the medical intensive care unit and those that required only a visit to the outpatient department.

Throughout the study period, all patients with SLE presenting with fever $\left(\geqslant 38^{\circ} \mathrm{C}\right)$ had at least an initial set of three blood cultures, a urine culture, and a chest radiograph. Common infections diagnosed in the cohort were defined as described by Mandell. ${ }^{8}$ Tuberculosis was always confirmed histologically or microbiologically. Finally, infections were characterised as community or nosocomially acquired, according to the time of their manifestation in relation to hospital admission.

\section{STATISTICAL ANALYSIS}

Statistical analyses were performed with Statview 4.5 (Abacus Concepts, Inc, Berkeley, California, USA). To identify risk factors predictive of infection, we first conducted univariate analyses to compare the different variables between infected and non-infected patients. The usual parametric methods, $\chi^{2}$ test with Yates's correction for small numbers, were used to compare qualitative variables. Student's $t$ test or Mann-Whitney U test, depending on the sample size, was used to compare quantitative variables expressed as mean (SD).

For patients who developed more than one infectious episode during the follow up period, a complementary analysis was conducted taking each episode into account.

The predictive power of variables (infection, SLE flare number, higher SLAM and SLEDAI during SLE evolution, type of organ involvement, and treatments) for mortality was analysed by comparing non-survivors with survivors 10 years after SLE diagnosis. Multivariate analysis of risk factors associated with the occurrence of infection or death used a stepwise logistic-regression model for the whole population; only items significantly associated with infection or increased risk of mortality according to the univariate analyses were included.

Spearman's correlation coefficient was used to establish correlations between SLAM and SLEDAI scores at diagnosis, and at the time of the most recent SLE flare preceding the infectious episode. $p \leqslant 0.05$ was considered significant.

\section{Results}

SUBJECTS

Eighty seven adults, 71 women and 16 men, were included in this study. Sixty two were white. Their median age at diagnosis was 33.7 years (range 16-80) and their median duration of follow up since SLE diagnosis was 9.4 years (range 1-37).

INFECTIOUS EPISODES

Of the 87 patients, $35(40 \%)$ had at least one infectious episode, while $52(60 \%)$ had none. Fifty seven infections were observed in these 35 patients: $22(63 \%)$ patients experienced one infectious episode and $13(37 \%)$ had at least two episodes.

SLAM and SLEDAI scores were correlated at all times studied (data not shown).

In $39 / 57(68 \%)$ cases of infection patients were admitted to the department of internal medicine, in eight $(14 \%)$ cases to the intensive care unit, and in $10(17 \%)$ cases were treated as out patients. The outpatient cases principally consisted of cutaneous infections (three abscesses caused by Staphylococcus spp, five caused by herpes zoster).

Forty six $(81 \%)$ infections were community acquired, and 11 (19\%) were nosocomial. Bacteria were responsible for $47 / 57(82 \%)$ of the episodes, with $40 \%(19 / 47)$ of them being infections of the lower respiratory tract.

Of the 16 patients with pneumonia, 10 were not microbiologically documented but presented with alveolar infiltrate(s) with raised serum $\mathrm{C}$ reactive protein levels. These infections always resolved favourably after treatment with conventional antimicrobial agents including a $\beta$-lactam antibiotic. Two patients with pneumonia had at least one positive blood culture for a penicillin susceptible strain of Streptococcus pneumoniae. The four patients who developed $S$ pneumoniae infections had not had a splenectomy and had normal plasma $\gamma$ globulin levels. None of them had been vaccinated against pneumococcus.

Seven patients receiving immunosuppressive treatment were given prophylaxis against Pneumocystis carinii (consisting of daily cotrimoxazole $(n=3)$ or monthly pentamidine isethionate in aerosol form $(n=4)$ ) because of profound and persistent lymphopenia $\left(<10^{9} / 1\right)$. None of the patients developed $P$ carinii pneumonia.

Only one of five patients who developed Mycobacterium tuberculosis infections had a documented primary infection during childhood and developed common pulmonary tuberculosis at SLE diagnosis. Two of three patients with positive sputum cultures had positive smears (for acid fast resistant bacilli), 
and two had characteristic histological features. Of the 52 non-infected patients, two who had histories of primary infection during childhood received prophylaxis against tuberculosis (a combination of isoniazid and rifampicin) for at least four months when corticosteroid treatment was started.

Bacteraemia was observed in 14 patients. Two cases were caused by Salmonella; one was complicated by lumbar spondylodiscitis.

Nosocomial infections represented 23\% (11/ 47) of all bacterial infections. Staphylococci predominated $(64 \%)$ but all strains were susceptible to meticillin. Two cases of $S$ aureus bacteraemia had secondary foci (spondylodiscitis and sacroiliitis in one case each). Venous catheters, one central and five peripheral, were the principal portals of entry.

All nine viral infections were caused by varicella zoster virus, but none was disseminated. The only invasive fungal infection, biopsy proven oesophagitis, was caused by Candida albicans.

Other infections were pyelonephritis, cholecystitis, acute sinusitis, and salpingitis.

RISK FACTORS FOR INFECTION

The basic characteristics of the infected and non-infected patients were similar (data not shown).

\section{Potential risk factors for infection}

At SLE diagnosis and during evolution, no differences were noted between the infected and non-infected groups in clinical involvement (renal, central nervous system, haematological), biological variables, and disease activity scores (data not shown).

The first infectious episode occurred after a mean of 4.5 (5.8) years. Table 1 gives the results of univariate and multivariate analyses of potential risk factors for infection.

\section{Risk factors at the time of the most recent SLE flare preceding the infectious episode} None of the clinical or biological variables (differential white blood cell counts, CD4 T lymphocyte counts, serum $\gamma$ globulin level, creatininaemia, inflammatory and immunological markers) predicted the occurrence of an infectious episode.

Table 1 Univariate analysis of potential risk ractors for infection in a single cohort of 87 adults with systemic lupus erythematosus (SLE)

\begin{tabular}{llll}
\hline Factor & $\begin{array}{l}\text { Infected } \\
(n=57)\end{array}$ & $\begin{array}{l}\text { Non-infected } \\
(n=52)\end{array}$ & $p$ Value \\
\hline $\begin{array}{l}\text { During SLE } \\
\quad \text { Total number of SLE flares }\end{array}$ & $2.6(1.5)$ & $1.8(1.1)$ & $<0.02$ \\
$\quad \begin{array}{ll}\text { Duration (years) } \\
\text { SLE glomerulonephritis }\end{array}$ & $11.8(6.6)$ & $8.4(8.5)$ & $<0.05$ \\
Treatments & $23 / 57(40 \%)$ & $10 / 52(19 \%)$ & $<0.05$ \\
$\quad$ Oral corticosteroids & $50 / 57(88 \%)$ & $38(73 \%)$ & $\mathrm{NS}$ \\
$\quad$ Oral corticosteroids (cumulative dose in g) & $30.9(40.3)$ & $8.5(15.4)$ & $<0.005$ \\
$\quad$ Intravenous corticosteroids & $27 / 57(47 \%)$ & $6(11 \%)$ & $<0.0001 \dagger$ \\
Intravenous corticosteroids (cumulative dose in g) & $1.3(2.2)$ & $0.4(1.4)$ & 0.01 \\
$\quad$ Immunosuppressive treatment & $32 / 57(56 \%)$ & $11(21 \%)$ & $<0.0005 \dagger$ \\
$\quad$ Pulse cyclophosphamide & $26 / 57(46 \%)$ & $9(17 \%)$ & $<0.003$ \\
$\quad$ Pulse cyclophosphamide (cumulative dose in $\mathrm{mg})$ & $5004(5603)$ & $1067(3397)$ & $<0.01$ \\
Plasmapheresis & $17 / 57(30 \%)$ & $4(8 \%)$ & $<0.01$
\end{tabular}

The infected group consisted of 35 adults with a total of 57 infectious episodes. Where applicable, values are mean $(\mathrm{SD})$.

†Retained as an independent risk factor for infection after multivariate analysis (intravenous corticosteroids: $\mathrm{p}<0.001$; immunosuppressive treatment: $\mathrm{p}<0.01$ )

NS = Not significant.
RISK FACTORS FOR DEATH

Ten patients died at a median time of 15.7 years (range 3-27; eight during the 10 years after SLE diagnosis), including two deaths attributed to infection (one from pneumococcal pelvioperitonitis and one from a gangrenous cholecystitis), four to severe SLE flares, two to cancer, and two to other causes not related to SLE. According to univariate and multivariate analyses, infection was the only independent risk factor for death 10 years after SLE diagnosis $(\mathrm{p}<0.01)$.

\section{Discussion}

Although risk factors for infection during SLE have been reported, ${ }^{13910}$ the analyses covered only short periods of time or concerned selected populations (patients in hospital or those requiring specific treatment).

Our study did not identify any risk factors for infection present at the time of SLE diagnosis. In addition, the disease activity initially, during SLE evolution, and not long before infection did not represent a risk factor for infection. Like others, ${ }^{10}$ we found that glomerulonephritis, but no other SLE complication, was a risk factor for infection.

Our univariate analysis showed corticosteroids to be a risk factor for infection and highlighted the importance of the cumulative dose. Interestingly, intravenous steroid treatment was retained as an independent risk factor, regardless of the time the infection occurred. Corticosteroids are known to decrease host defences by their multiple immunological effects and thus to favour infections. ${ }^{3} 10$ Oral corticosteroids are routinely prescribed during SLE flares, but no standardised consensus regimen has been established. ${ }^{11}$ Intravenous corticosteroids are given for severe SLE flares because of their presumed rapid efficacy, which has never been confirmed. Interestingly, in another study, a prior history of intravenous steroid treatment was found to be a risk factor for tuberculosis in patients with various rheumatic diseases. ${ }^{12}$ Thus intravenous steroids should be used cautiously in patients with SLE. Over the past decade, cyclophosphamide has been prescribed to treat life threatening manifestations of SLE. It has been advocated exclusively for diffuse proliferative lupus glomerulonephritis and prevents progression to renal failure, but its complications, notably infections, are becoming more common. ${ }^{13}$ Several studies found cyclophosphamide, and other immunosuppressive drugs, to be responsible for more infections, ${ }^{13}$ because they induce leucopenia. ${ }^{3}$

A predominance of community acquired bacterial infections, especially those caused by $S$ pneumoniae, was found here. Because of this incidence and the potential severity of $S$ pneumoniae infections, treatment with antibiotics active against this bacterium should be started promptly.

This series, contrasting with results obtained by others, included numerous cases of tuberculosis. ${ }^{1{ }^{10}}$ All these patients had been taking oral corticosteroids before developing tuberculosis. $^{312}$ It may thus be advisable to prescribe 
prophylaxis against tuberculosis at the onset of corticosteroid treatment in high risk groups such as migrant populations, those in contact with infected individuals, those with a history of tuberculosis during childhood, or when the chest radiograph shows residual tuberculosis. The clinical value of the tuberculin skin test remains controversial. ${ }^{12}$ Indeed, in a prospective study, 30 patients with rheumatic disease developed tuberculosis infection after the start of steroid treatment, but only eight of them had a previous positive tuberculosis skin test. ${ }^{12}$

Concerning opportunistic infections, we observed only a $C$ albicans oesophagitis. No other fungal infection or parasitic diseases were noted during SLE evolution or at autopsy. ${ }^{314}$ Although, we had no cases of $P$ carinii pneumonia during the entire study period, others have reported the occurrence of this opportunistic infection. ${ }^{315}$ Thus cotrimoxazole prophylaxis may be warranted in heavily immunosuppressed patients with lymphopenia.

Our study also clearly shows that infection is an independent risk factor for death at 10 years of SLE evolution, although two deaths can be directly attributed to infection.

Because of the independent role of immunosuppressive agents, particularly intravenous steroids, in the occurrence of infection during SLE and their independent effect on the long term prognosis, it is imperative to find a way to identify patients who should be targeted for such treatment. To avoid unacceptably high morbidity and mortality, careful follow up of patients with SLE is recommended, as infection, mainly of bacterial origin, can occur at any time during evolution of the disease and cannot be predicted by SLE activity.
We thank Janet Jacobson for reviewing the English.

1 Pryor BD, Bologna SG, Kahl LE. Risk factors for serious infection during treatment with cyclophosphamide and high-dose corticosteroids for systemic lupus erythematohigh-dose corticosteroids for systemic lup
sus. Arthritis Rheum 1996;39:1475-82.

2 Rosner S, Ginzler EM, Diamond HS, Weiner M, Schlesinger $\mathrm{M}$, Fries JF, et al. A multicenter study of outcome in systemic lupus erythematosus. II. Causes of death. Arthriis Rheum 1982;25:612-17.

3 Iliopoulos AG, Tsokos GC. Immunopathogenesis and spectrum of infections in systemic lupus erythematosus. Semin Arthritis Rheum 1996;25:318-36.

4 Cervera R, Khamashta MA, Font J, Sebastiani CD, Gill A, Lavilla P. Morbidity and mortality in systemic lupus erythematosus during a 5-year period: a multicenter prospective study

5 Tan EM, Cohen AS, Fries JF, Masi AT, McShane DJ, RothTield DJ, et al. The 1982 revised criteria for the classification of systemic lupus erythematosus. Arthritis Rheum 1982; of systemic

6 Wallace DJ, Hahn BH. In: Dubois's lupus erythematosus. 5th ed. Baltimore: Williams and Wilkins, 1997:627-1065.

7 Stuck AE, Minder CE, Frey FJ. Risk of infectious complications in patients taking glucocorticosteroids. Rev Infect Dis 1989;11:954-63.

8 Mandell GL, Bennett JE, Dolin R. In: Principles and practice of infectious diseases. 5th ed. Philadelphia: Churchill Livingstone, 2000.

9 Nived O, Sturfelt G, Wollheim F. Systemic lupus erythematosus and infection: a controlled and prospective study including an epidemiological group. QJM 1985;55:27187.

10 Ginzler E, Diamond H, Kaplan D, Weiner M, Schlesinger $M$, Seleznick $M$. Computer analysis of factors influencing frequency of infection in systemic lupus erythematosus. frequency of infection in system
Arthritis Rheum 1978;21:37-44

11 Corzillius M, Bae SC. Methodological issues of corticosteroid use in SLE clinical trials. Lupus 1999;8:692-7.

12 Hernandez-Cruz B, Sifuentes-Orsonio J, Ponce-de-Leon RS, Ponce-de-Leon Garduno A, Diaz-Jouanen E. Mycobacterium tuberculosis infection in patients with systemic rheumatic diseases. A case series. Clin Exp Rheumatol 1999;17:289-96.

13 Ortmann RA, Klippel JH. Update on cyclophosphamide for systemic lupus erythematosus. Rheum Dis Clin North Am 2000;26:363-375.

14 Hellmann DB, Petri M, Whiting-O'Keefe Q. Fatal infections in systemic lupus erythematosus: the role of opportunistic organisms. Medicine (Baltimore) 1987;66: opportuni $341-8$.

15 Godeau B, Coutant-Perrone V, Lê Thi Huong D, Guillevin L, Magadur G, De Bandt M, et al. Pneumocystis carinii pneumonia in the course of connective tissue disease. J Rheumatol 1994;21:246-51. 